This is the last draft sent to the Editorial by the authors of the article:

M. GÓMEZ, S. F. MEDINA, J. I. CHAVES
"Static Recrystallisation of Austenite in a Medium-Carbon Vanadium
Microalloyed Steel and Inhibition by Strain-Induced Precipitates"
Materials Science Forum
Vol. 550 (2007), Pages: $417-422$
DOI: $10.4028 / 0-87849-434-0.417$
ISSN: $0255-5476$

To be published in Digital.CSIC, the Institutional Repository of the Spanish National Research Council (CSIC)

See more papers from the authors on:

$\underline{\text { http://digital.csic.es }}$

http://www.researcherid.com/rid/B-7922-2008 


\title{
Static Recrystallization of Austenite in a Medium-Carbon Vanadium Microalloyed Steel and Inhibition by Strain-Induced Precipitates
}

\author{
M. Gomez ${ }^{1,2, a}$, S. F. Medina ${ }^{1, b}$ and J. I. Chaves ${ }^{1, c}$ \\ ${ }^{1}$ National Centre for Metallurgical Research, CENIM, Dept. of Physical Metallurgy, Avda. Gregorio \\ del Amo 8, Madrid, 28040, Spain. \\ ${ }^{2}$ University of Pittsburgh, Basic Metals Processing Research Institute (BAMPRI), Department of \\ Materials Science and Engineering, Pittsburgh, PA 15261 USA. \\ amgomez@cenim.csic.es, bsmedina@cenim.csic.es, ’jichaves@cenim.csic.es
}

Keywords: vanadium microalloyed steel, static recrystallization of austenite, strain induced precipitation, driving and pinning forces.

\begin{abstract}
The austenite static recrystallization kinetics at several temperatures and the recrystallization-precipitation-time- temperature (RPTT) diagrams of a medium-carbon vanadium microalloyed steel have been determined for a strain $\varepsilon=0.35$. Unlike many other studies carried out previously on $\mathrm{V}$ microalloyed steels, the recrystallized fraction against time curves showed the formation of a double plateau that indicates two stages of inhibition of recrystallization due to the formation of different types of strain induced precipitates. This work makes use of transmission electron microscopy to study the nature and size distribution of these precipitates capable of inhibiting recrystallization. The values of driving and pinning forces for static recrystallization are calculated and an analysis of the relationship between the net balance of these forces, the precipitation state and the progress or inhibition of the recrystallization is accomplished. A value of driving force that decreases as recrystallized fraction grows during isothermal holding time is estimated and helps to interpret the behavior of austenite after deformation.
\end{abstract}

\section{Introduction}

The static recrystallization of microalloyed steels after hot deformation can be divided into two stages: before and after the start of induced precipitation. In the first stage the curves of recrystallized fraction against time $\left(\left(X_{\mathrm{a}}(\mathrm{t})\right)\right.$ are similar to those for non microalloyed steels and in the second stage the curves display a plateau, caused by the formation of precipitates which inhibit recrystallization. $[1,2]$. Before and after the plateau, the curve $\left(\left(X_{\mathrm{a}}(\mathrm{t})\right)\right.$ obeys Avrami's law and this together with the knowledge of the times for the beginning and the end of the plateaus of inhibition of recrystallization makes it possible to easily draw recrystallization-precipitation-time-temperature (RPTT) diagrams where the interaction between these two processes is clearly observed $[3,4]$. The limit between the two stages comes at a point known as Static Recrystallization Critical Temperature (SRCT), which is the temperature where the inhibition of recrystallization begins as a consequence of precipitation.

The influence of vanadium (dissolved in the austenite or precipitated) on static recrystallization is weaker than that observed for niobium, but it is still significant, as values of activation energy $Q$ and SRCT show [4]. This work focuses on the static recrystallization kinetics of a medium carbon $\mathrm{V}$-microalloyed steel and presents a study of austenite microstructure and precipitation state. Additionally, the competition between the processes of recrystallization of austenite after deformation at high temperature and retardation of grain boundary motion due to the effect of fine particles is quantified with the help of expressions for the driving and pinning forces found in the literature [5].

Driving and pinning forces for recrystallization. According to the model of strain induced grain boundary migration [6], recrystallization driving force $F_{\mathrm{R}}$ comes from the difference in dislocation density across the moving boundary separating two adjacent grains. $F_{\mathrm{R}}$ can be expressed as: 


$$
F_{R}=\mu b^{2} \Delta \rho / 2 \text {. }
$$

where $\mu$ is the shear modulus $\left(4 \cdot 10^{4} \mathrm{MN} / \mathrm{m}^{2}\right.$ for austenite), $b$ the Burgers vector $\left(2 \cdot 10^{-10} \mathrm{~m}\right)$ and $\Delta \rho$ the change in dislocation density, related to the rise in flow stress during work hardening $\Delta \sigma$ [7] by:

$$
\Delta \sigma=0.2 \mu b \sqrt{\Delta \rho}
$$

The recrystallization pinning force $F_{\mathrm{P}}$ arises from the area of boundary removed by precipitates [8]. For a population of $N_{\mathrm{s}}$ particles per unit area of grain boundary having a mean radius $r, F_{\mathrm{P}}$ will be [9]:

$$
F_{p}=4 r \gamma N_{s}
$$

where $\gamma$ is the interfacial energy per unit area of boundary $\left(0.8 \mathrm{~J} / \mathrm{m}^{2}\right.$ in austenite). Knowing that the number of particles per unit volume $N_{\mathrm{v}}$ and the volume fraction $f$ obey:

$$
N_{v}=\frac{3 f}{4 \pi r^{3}} \text {. }
$$

three different models to calculate $F_{\mathrm{P}}$ have been proposed, depending on the $N_{\mathrm{s}}-N_{\mathrm{v}}$ relationship: the rigid boundary, the flexible boundary and the sub-boundary model. In this study the flexible boundary model (FBM) was adopted because it frequently provides the most reasonable results $[5,10]$. This model considers that an infinitely flexible boundary interacts with every particle in the 3 -D array until it is fully pinned and leads to this expression.

$$
F_{p}=\frac{3 \gamma f^{2 / 3}}{\pi r}
$$

The volume fraction was calculated from the data of precipitates collected in the TEM (mean value of diameters $d$, standard deviation of size distribution $s$ and number density per unit area $N_{p}$ ) [11]:

$$
f=\frac{\pi}{6} N_{p}\left(d^{2}+\sigma^{2}\right)
$$

\section{Experimental Procedure}

The steel studied, whose composition is shown in Table 1, was manufactured by Electroslag Remelting (ESR). In order to study the static recrystallization kinetics of the austenite by means of the "Back Extrapolation" method [12], double deformation tests were carried out in a computer-controlled hot torsion machine. Torsion magnitudes (torque, no. of revolutions) and equivalent magnitudes (stress, strain) were related according to the Von Mises criterion [13]. The specimens were austenitized at $1200^{\circ} \mathrm{C}$ for $10 \mathrm{~min}$. These reheating conditions were enough to completely dissolve the vanadium precipitates, as solubility temperatures calculated for nitrides and carbides were equal to $1095{ }^{\circ} \mathrm{C}$ and $844{ }^{\circ} \mathrm{C}$, respectively [14]. However, a certain amount of undissolved $\mathrm{TiN}$ can be expected to remain after reheating, as the calculated temperature for complete solution of this type of precipitate is $1385{ }^{\circ} \mathrm{C}$. The samples were then cooled and two deformation steps were applied at several temperatures between $850{ }^{\circ} \mathrm{C}$ and $1000{ }^{\circ} \mathrm{C}$. The value of equivalent strain applied in each step was 0.35 , lower than the critical strain which leads to the start of dynamic recrystallization, and the strain rate was equal to $3.628 \mathrm{~s}^{-1}$. Isothermal holding time between 
steps was varied between 0.5 and $1000 \mathrm{~s}$.

In order to verify the accuracy of the method of determination of the recrystallized fraction, to observe the advance/inhibition of recrystallization and to study the influence of interpass time on microstructure, precipitation state and pinning forces, five samples were water-quenched after applying a deformation step at $875^{\circ} \mathrm{C}$ and carrying out an isothermal holding period of $2,7,18,70$ and $700 \mathrm{~s}$. Microstructures were observed on a longitudinal surface and the characteristics of precipitates were determined by transmission electron microscopy (TEM), using the carbon extraction replica technique.

Table 1. Chemical composition of steel studied [mass \%].

\begin{tabular}{|c|c|c|c|c|c|c|c|c|c|c|c|}
\hline $\mathrm{C}$ & $\mathrm{Si}$ & $\mathrm{Mn}$ & $\mathrm{P}$ & $\mathrm{S}$ & $\mathrm{Al}$ & $\mathrm{Cu}$ & $\mathrm{Cr}$ & $\mathrm{V}$ & $\mathrm{Ti}$ & $\mathrm{N}$ & $\mathrm{O}$ \\
\hline 0.46 & 0.26 & 1.30 & 0.027 & 0.016 & 0.008 & 0.007 & 0.22 & 0.068 & 0.003 & 0.0250 & 0.0035 \\
\hline
\end{tabular}

\section{Results and Discussion}

Static recrystallization kinetics. First of all, the initial austenite grain size after reheating at $1200{ }^{\circ} \mathrm{C}$ was determined according to ASTM standard E-112, being found a value of $165 \mu \mathrm{m}$. Fig. 1 shows the relationship between recrystallized fraction and time. At higher temperatures $\left(1000,900{ }^{\circ} \mathrm{C}\right)$ the recrystallization progresses continuously until completion, but at lower temperatures a double plateau is formed, indicating two time intervals where the recrystallization of austenite is inhibited. Previous studies have verified by means of differential thermal analysis (DTA) [3] and lattice parameter comparison [15] that this phenomenon results from the formation of two different types of precipitates having different stoichiometries and similar solubility temperatures. A novel aspect in this work is that this double plateau has usually been experimentally found [16] or simulated [17] on $\mathrm{Nb}$ steels, but never on V-steels. The RPTT diagram of Fig. $1 \mathrm{~b}$ shows the formation of two $P_{\mathrm{s}}$ and $P_{\mathrm{f}}$ curves and two values of SRCT, corresponding to two separated stages of precipitation.

a)

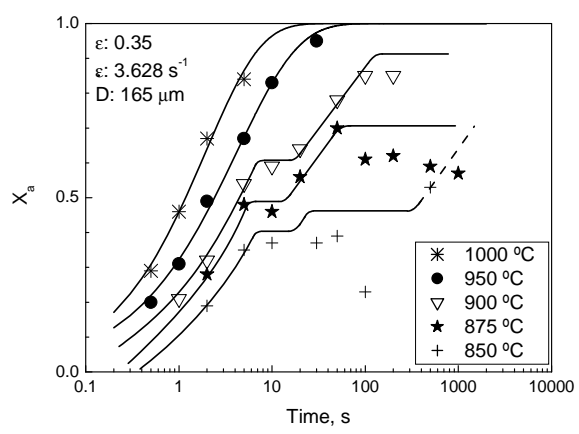

b)

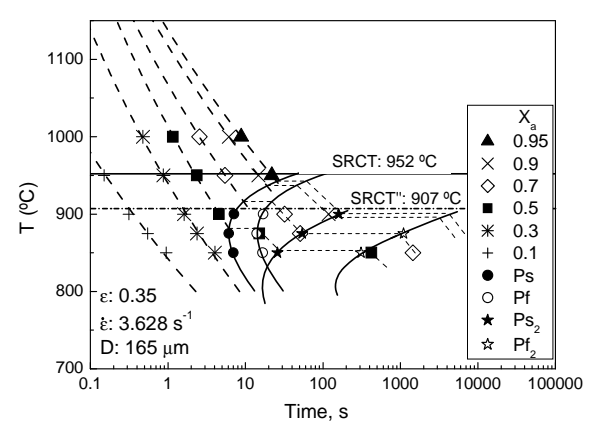

Fig. 1. a) Curves of recrystallized fraction of austenite against time, $\varepsilon=0.35$. b) RPTT diagram.

Austenite microstructure and precipitation state. Figs. $2 a$ and $2 b$ show two examples of deformed and substantially recrystallized structures, respectively. The difference between the calculated and observed recrystallized fractions in the five quenched samples was always below $7 \%$.

The samples were subsequently observed by TEM to study the precipitation state. In all the samples (Ti, V) nitride or carbonitride particles were found. The precipitation starts in the early stages after deformation, as the sample quenched after $2 \mathrm{~s}$ already shows particles (Fig. 3a). There is a low amount of precipitates and these concentrate on defects, but as isothermal holding time after deformation increases, they are more frequent and homogeneously distributed (Fig. 3b). Coalescence is observed for the longest times, but before the end of precipitation. Size distribution of particles varies among the samples and that strongly influences the pinning effect exerted by the precipitates. The most frequent particle size is generally about $6 \mathrm{~nm}$ (Figs. 4a to 4e), but the mean precipitate size grows with time (Fig. 5), due to a rising presence of coarse particles (wider distribution, higher standard deviation). The accumulated frequency plot of Fig. $4 \mathrm{f}$ shows that for shorter times there is a generalized nucleation and growth of particles as time passes (the three first curves take similar shape 
but they move to bigger sizes). After $2 \mathrm{~s}$, the precipitates are very fine but recrystallization is not yet stopped. Therefore, the appearance of the first plateau at $\mathrm{t} \approx 7 \mathrm{~s}$ should come from the increase in the volume fraction of these very fine particles. The particles keep growing and lose part of their capability to retard recrystallization, so the first plateau ends. However, Fig $4 \mathrm{~d}$ shows that the fourth sample $(t=70 \mathrm{~s})$ presents a marked maximum corresponding to fine sizes $(4-8 \mathrm{~nm})$ which indicates a second stage of nucleation of new particles that would cause an increase in the pinning effect and the start of the second plateau of inhibition of recrystallization. In the last curve, a coarsening of the particles that existed at $70 \mathrm{~s}$ is observed. Near $15 \%$ of particles is coarser than $50 \mathrm{~nm}$ (Fig. 4b) and the curve of accumulated frequency moves to the right (Fig. 4f) so retardation of recrystallization by particles will get weaker.

Lattice parameters of a large population of particles were determined in each sample. The average values slightly increased until reaching a maximum $(0.420 \mathrm{~nm})$ for the sample quenched after $70 \mathrm{~s}$ and then decreased. It is known that carbides and Ti compounds have higher lattice parameters than nitrides and V compounds, respectively [18]. Besides, TiN usually precipitates at higher temperatures than VN and heterogeneous nucleation is likely to occur. Therefore this evolution can indicate that the precipitate stoichiometry is initially a $\mathrm{N}$ rich $(\mathrm{Ti}, \mathrm{V})$ carbonitride and it changes with time to a C-richer carbonitride with increasing V content, which would have caused the second plateau.

a)

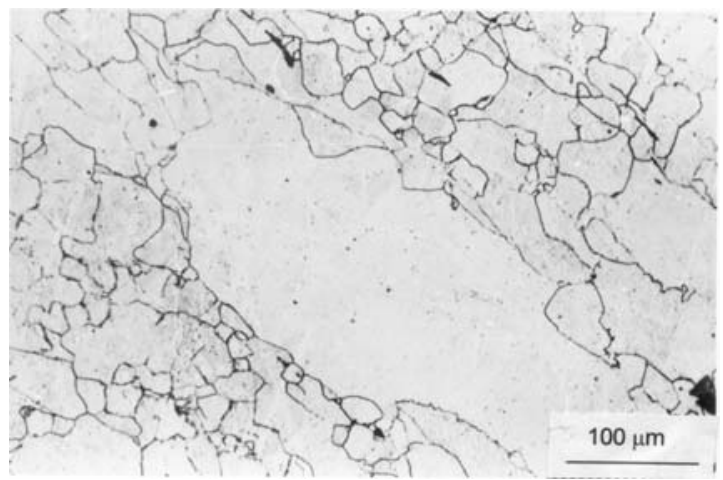

b)

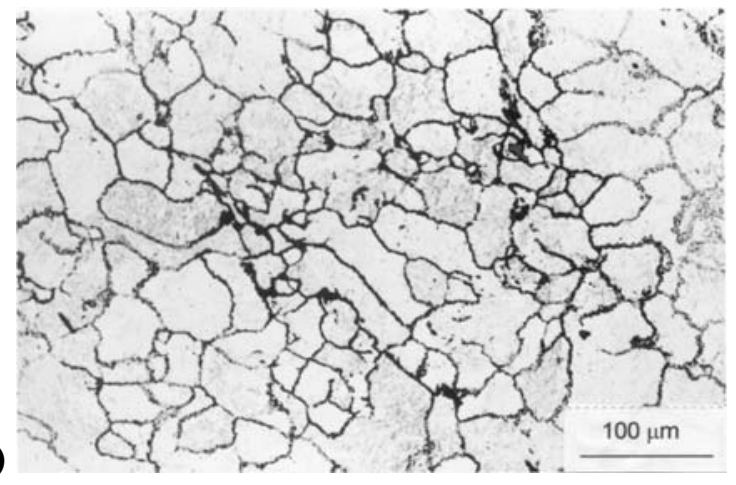

Fig. 2. Austenite microstructure of samples quenched after deformation at $875{ }^{\circ} \mathrm{C}$ followed by isothermal holding $t_{\mathrm{rec}}$. a) $t_{\mathrm{rec}}=2 \mathrm{~s}$; b) $t_{\mathrm{rec}}=700 \mathrm{~s}$.

a)

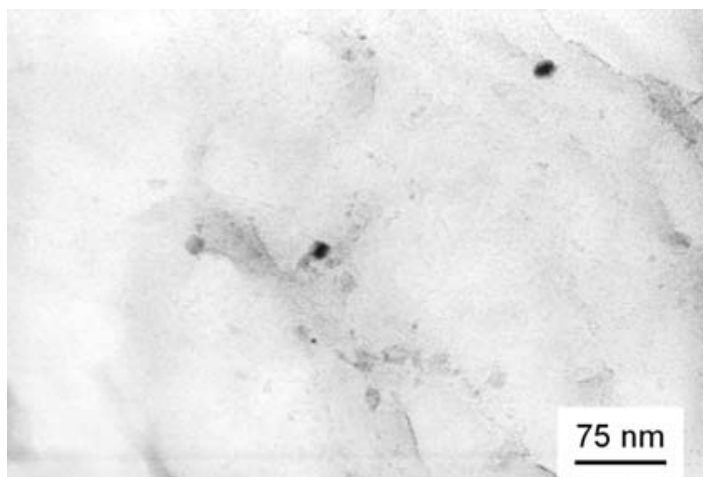

b)

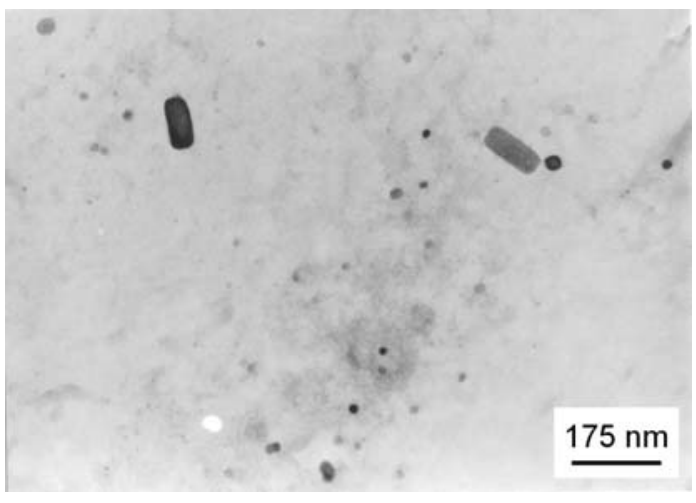

Fig. 3. TEM images showing the precipitation state of samples quenched after deformation at $875^{\circ} \mathrm{C}$ followed by isothermal holding $t_{\mathrm{rec}}$. a) $t_{\mathrm{rec}}=2 \mathrm{~s}$; b) $t_{\mathrm{rec}}=700 \mathrm{~s}$.

Driving and pinning forces for recrystallization. Fig. 6 presents the values of the driving and pinning forces of recrystallization calculated with Eqs. 1 to 6. Different criteria can be chosen to estimate the dislocation density $\Delta \rho$ from the maximal or the Mean Flow Stress (MFS) [5]. Besides, the value of the driving force $F_{R}$ calculated from Eqs. 1 and 2 should be taken as the power of recrystallization contained in the internal structure of the austenite just after a deformation step. This potential $F_{\mathrm{R}}$ can be reduced by the effect of recovery previous to recrystallization [19]. More 
importantly, as long as time passes and the austenite recrystallizes there will be a continuous reduction in $\Delta \rho$ leading to a decrease in $F_{R}$. The real dislocation density $\left(\Delta \rho_{\mathrm{A}}\right)$ will be then a function of recrystallized fraction $\left(X_{\mathrm{a}}\right)$ by [20]:

$$
\Delta \rho_{A}=\left(1-X_{a}\right) \Delta \rho+X_{a} \rho_{0}
$$

where $\Delta \rho$ and $\rho_{0}$ are the density of dislocations in the strained and completely recrystallized austenite, respectively $\left(\rho_{0}=2 \cdot 10^{12} \mathrm{~m}^{-2}\right)$. This expression allows us to determine a $F_{\mathrm{R}}$ which is a function of the recrystallized fraction and decreases with time, except during the plateaus of inhibition of recrystallization, where it is constant, as Fig. 6 shows.

The evolution of the balance $\left(F_{\mathrm{R}}-F_{\mathrm{P}}\right)$ shown in Fig. 6 has a reasonable agreement with the observed behavior: for the shortest times, the precipitates are very fine but not abundant enough to stop recrystallization, so $F_{\mathrm{P}}$ takes values below $F_{\mathrm{R}}$. As isothermal holding time increases, volume fraction of fine particles grows and $F_{\mathrm{P}}$ value raises and surpasses $F_{\mathrm{R}}$. In this sense, the calculation of the decreasing $F_{\mathrm{R}}$ proposed here helps to explain better why the first plateau takes place. Later, $F_{\mathrm{P}}$ reaches a maximum corresponding to the beginning of the second plateau (much longer than the first one) which would correspond to the optimal conditions for recrystallization inhibition. For very long times, coarsening of particles makes $F_{\mathrm{P}}$ to decrease again below $F_{\mathrm{R}}$. The blockage of recrystallization finishes and the curves of Fig. 1 will take again an Avrami trend. Nevertheless, the evolution of $F_{\mathrm{P}}$ is complex and for example the trend between the two plateaus (where recrystallization momentarily progresses) is still unclear.

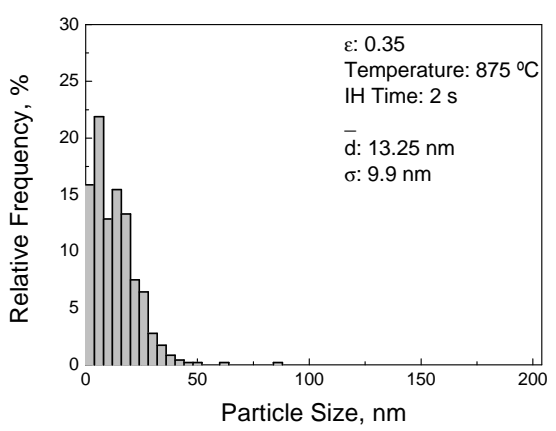

a)

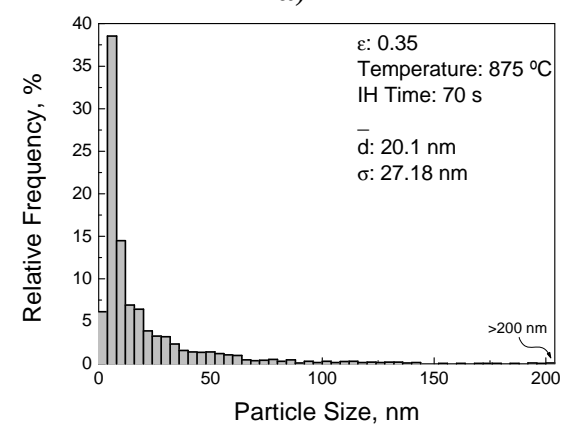

d)

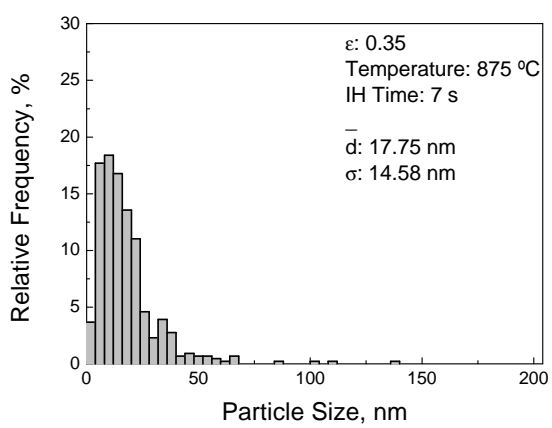

b)

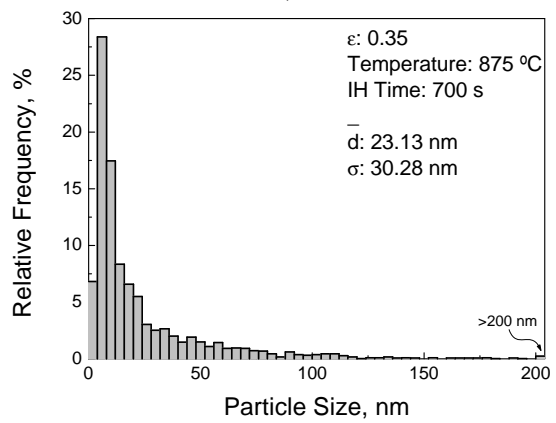

e)

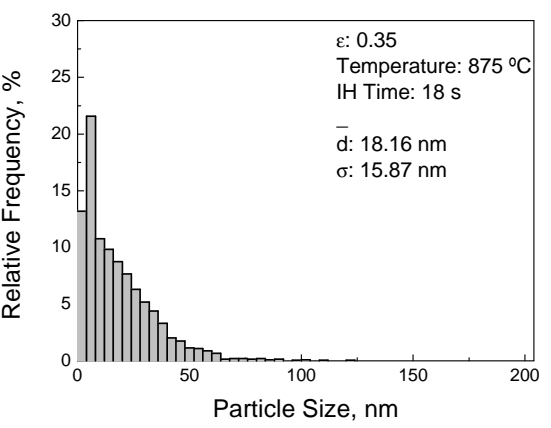

c)

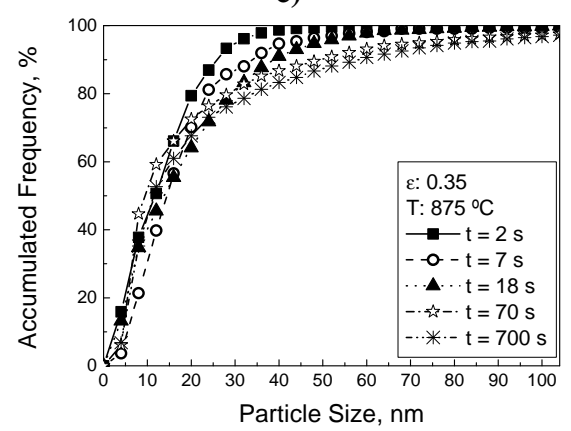

f)

Fig. 4. a) to e) Frequency distribution of sizes of particles found after one deformation at $875{ }^{\circ} \mathrm{C}$ and isothermal holding of 2, 7, 18, 70 and $700 \mathrm{~s}$, respectively. f) Accumulated percentage of particles against their size.

\section{Summary}

The two plateaus of inhibition of recrystallization found in the curves of recrystallized fraction against time for the $\mathrm{V}$-microalloyed steel studied originate from the pinning forces exerted by two successive precipitations of $(\mathrm{Ti}, \mathrm{V})$ carbonitrides whose stoichiometry tends to higher $\mathrm{V}$ and $\mathrm{C}$ contents as time after deformation increases. Moreover, a driving force which is a function of 
recrystallized fraction has been determined and assists in the interpretation of recrystallization inhibition by particles.

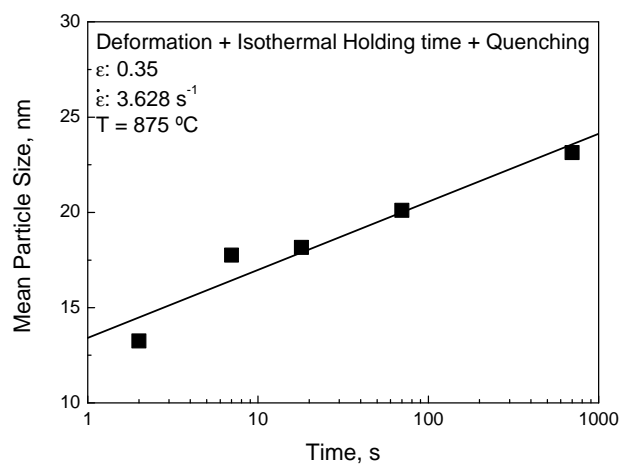

Fig. 5. Evolution of mean particle size against isothermal holding time after deformation.

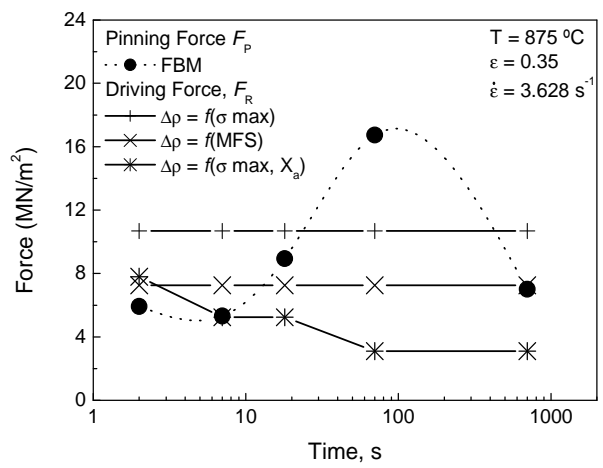

Fig. 6. Evolution of recrystallization driving $\left(F_{\mathrm{R}}\right)$ and pinning forces $\left(F_{\mathrm{P}}\right)$ against the time after deformation. Values obtained by taking different criteria to estimate dislocation density are compared

\section{References}

[1] F. H. Samuel. S. Yue, J. J. Jonas and B. A. Zbinden: ISIJ Int., Vol. 29 (1989), p. 878.

[2] S. F. Medina, A. Quispe. ISIJ Int., Vol. 41 (2001), 7, p. 774.

[3] S. F. Medina, A. Quispe, P. Valles and J. L. Baños. ISIJ Int. Vol. 39 (1999), 9, p. 913.

[4] A. Quispe, S. F. Medina, P. Valles. ISIJ Int. Vol. 37 (1997), 8, p. 783.

[5] M. Gómez, S.F. Medina. Mater. Sci. Forum Vol. 500-501 (2005) p. 147.

[6] A. Beck and P.R. Sperry: Journal of Applied Physics, Vol. 25, (1950), p. 150

[7] A.S. Keh: Direct Observations of Imperfections in Crystals (J.B. Newkirk and J.H. Wernick, eds. Wiley-Interscience, New York, 1962), p. 213.

[8] C.S. Smith: Trans. AIME, 175, 15, (1948), p. 15. (Ref. 24).

[9] T. Gladman: Proc. Royal Society, 294 (1966), p. 298.

[10]. M. Arribas, B. López, J.M. Rodriguez-Ibabe. Mater. Sci. Forum Vol. 500-501 (2005) p.131.

[11] M.F. Ashby, R. Ebeling: Trans. AIME, Vol. 236, (1966), p. 1396.

[12]H.L. Andrade, M.G. Akben and J.J. Jonas: Metall. Trans. Vol. 14A (1983), p. 1967

[13]A. Faessel: Rev. Metall. CIT, 33, 4 (1976), p.875.

[14]E.T. Turkdogan: Trans. ISS, 3, 5 (1989), p. 61.

[15] M. Gómez, S.F. Medina, P. Valles and A. Quispe. Mater. Sci. Forum Vol. 480-481 (2005) p. 489.

[16] S. G. Hong, K. B. Kang and C. G. Park. Scripta Materialia, Vol. 46 (2002), 2, p. 163

[17]H.S. Zurob, C.R. Hutchinson, Y. Brechet, G.R. Purdy. Mater. Sci. Eng. 382A (2004) p. 64.

[18] W. B. Pearson. A Handbook of lattice spacings and structures of metals and alloys (Pergamon Press 1958).

[19]E. J. Palmiere, C. I. Garcia, A. J. DeArdo. ISS of A.I.M.E., Warrendale, PA (1992) p. 113.

[20]B. Dutta, E.J. Palmiere and C.M. Sellars: Acta Mater. Vol. 49 (2001), p. 785. 